\title{
Effectiveness of Hyperbaric Oxygenation Versus Normobaric Oxygenation Therapy in Carbon Monoxide Poisoning: A Systematic Review
}

\author{
Sebastian Casillas ${ }^{1}$, Antonio Galindo ${ }^{1}$, Luis A. Camarillo-Reyes ${ }^{1}$, Joseph Varon ${ }^{2,} 3$, Salim R. Surani ${ }^{4,5,6}$ \\ 1. Research, Dorrington Medical Associates, Houston, USA 2. Critical Care, United General Hospital, Houston, USA 3. \\ Critical Care, University of Texas Health Science Center, Houston, USA 4. Internal Medicine, Texas A\&M Health Science \\ Center, Temple, USA 5. Internal Medicine, Corpus Christi Medical Center, Corpus Christi, USA 6. Internal Medicine, \\ University of North Texas, Dallas, USA
}

Corresponding author: Joseph Varon, jvaron@roamer.net

\begin{abstract}
Carbon monoxide (CO) is a gas product of combustion, considered highly poisonous. Prolonged CO exposure is responsible for more than half of fatal poisonings and is also one of the leading causes of poisoning in Western countries.

We aimed to compare the effectiveness of therapy with hyperbaric oxygen (HBO) versus normobaric oxygen (NBO) in the setting of carbon monoxide poisoning (COP). We independently searched the National Library of Medicine's Medline (PubMed ${ }^{\mathrm{TM}}$ ), ScienceDirect ${ }^{\mathrm{TM}}$, and Scielo ${ }^{\mathrm{TM}}$ for any relevant studies published from 1989 to 2017, using the following keywords: hyperbaric therapy, hyperbaric oxygenation, normobaric therapy, carbon monoxide poisoning, carboxyhemoglobin, Haldane effect. We analyzed the studies that suggested the effectiveness of $\mathrm{HBO}$ or NBO. Also, we searched for studies related to COP; including history, epidemiology (risk factors, incidence, demographics), pathophysiology, clinical manifestations, diagnosis, and treatment.
\end{abstract}

Sixty-eight articles were found, sixteen of which dealt with either $\mathrm{HBO}$ or NBO or both. Twelve suggested $\mathrm{HBO}$ as the treatment of choice in $\mathrm{COP}$; four studies indicated that $\mathrm{NBO}$ was an adequate treatment due to its cost-effectiveness and availability in the emergency department (ED).

HBO has been shown in several studies to be effective in moderate to high-risk COP situations, being the therapy of choice to avoid sequelae, especially neurologically. NBO can be considered as a reasonable alternative due to its cost-effectiveness. The availability and understanding of different therapeutic interventions are critical in the management of patients with COP in ED and the Critical Care unit.

Received 09/05/2019

Review began 09/09/2019

Review ended 10/05/2019

Published 10/15/2019

๑) Copyright 2019

Casillas et al. This is an open access article distributed under the terms of the Creative Commons Attribution License CC-BY 3.0., which permits unrestricted use, distribution, and reproduction in any medium, provided the original author and source are credited.
Categories: Internal Medicine, Medical Education, Healthcare Technology

Keywords: carbon monoxide poisoning, hyperbaric oxygen therapy, normobaric oxygen therapy, haldane effect

\section{Introduction And Background}

Carbon monoxide (CO) is a colorless gas, with no smell or taste and is a product of combustion [1]. This gas is considered highly poisonous [2]. Common sources for CO include malfunctioning heating systems, the exhaust of internal combustion engines, incomplete combustion of fuels, and inhaled smoke [3,4]. High environmental concentrations of $\mathrm{CO}$ can be found in areas with poor ventilation and may cause signs and symptoms of intoxication [5,6]. Prolonged CO exposure is responsible for more than half of fatal poisonings, and is also one of the leading causes of poisoning in Western countries with several visits to the emergency department (ED) each year, just in the United States [5,7,8]. Hyperbaric oxygen (HBO) and normobaric oxygen (NBO) have been suggested as the key therapeutic options with several controversies presented between different studies regarding methods, the number of patients, absolute atmospheres (ATA) given during HBO therapy and overall clinical benefit [9]. One of the most widely quoted papers is the study by Raphael and colleagues who performed a randomized trial, assessing the controversial and debated topic regarding therapeutic advantages and indications in the treatment of $\mathrm{CO}$ poisoning with $\mathrm{HBO}$, compared with the classical paramount management with NBO [10]. Six hundred and twenty-nine adult patients were included in this study; inclusion criteria consisted of CO poisoning (COP) within 12 hours prior to admission. The studied population was divided into four groups based on the level of consciousness for which $\mathrm{HBO}$ and NBO were administered alone or in combination. The study concluded a reduction in neurological sequelae in those patients that sustained a loss of consciousness and attributed this effect to the wider use of $\mathrm{HBO}[10]$.

The clinical manifestations of CO poisoning are highly unspecific; these include headache, myalgia, dizziness (in severe exposures, confusion), loss of consciousness, and death [4,7]. CO binds to hemoglobin 200-230 times more effectively than oxygen. This causes a leftward shift in the oxygen-hemoglobin 
dissociation curve, decreasing oxygen delivery to the tissues; the resulting tissue hypoxia has been proposed as the main mechanism of CO toxicity. CO additionally affects the cerebral perfusion through altered vasoactivity and neuronal responses independent of hypoxic stress [1]. The resulting generation of carboxyhemoglobin produces a left shift dissociation on the oxygen-hemoglobin curve. This was, first described by Claude Bernard in 1865 [8].

Prolonged CO exposure increases cytosolic heme levels, which leads to oxidative stress and binds to platelet heme protein and cytochrome c oxidase, which interrupts cellular respiration, causing increased reactive oxygen species generation leading to neural necrosis and apoptosis [7]. Given its highly unspecific clinical presentation, $\mathrm{CO}$ poisoning remains one of the most challenging medical conditions to diagnose, the key element to this puzzle remains on the high level of suspicion that the physician must have based on a detailed history, including symptom duration and correlation with environmental CO exposure [11,12]. Current methods to properly diagnose CO poisoning reside in the measurement of carboxyhemoglobin $(\mathrm{COHb})$; it is a useful diagnostic method but is considered a weak indicator of the severity of poisoning, a consequence of the delay of the blood assay and timing of poisoning occurrence $[9,13]$. The purpose of this article is to systematically review these two therapeutic interventions and compare their effectiveness.

\section{History}

The Greek and Roman empires made the first documented registry of CO application; both civilizations were familiar with this gas toxicity and found ways to apply its properties in the punishment of criminals. It was in 1880 when William Cruikshank described the main chemical properties of CO. In the middle of the 19th century, French physiologist Claude Bernard recognized that CO caused hypoxia by direct interaction with hemoglobin, and at the end of the century, Haldane studied the relationship between the high partial pressure of oxygen and its therapeutic effect on hemoglobin and CO [14].

\section{Epidemiology}

Prolonged CO exposure represents one of the most common causes of unintentional intoxication worldwide $[15,16]$. It is mainly seen in a residential setting in the US and western countries. The high occurrence is confirmed by the Centers for Disease Control and Prevention (CDC) with an incidence of 23.2/million population per year and nearly 15,000 emergency department visits and 500 deaths each year in the United States alone [17]. Most of the CO exposures occur at home, and mostly involve females, children aged $\leqslant 17$ years, and adults aged 18-44 years. On analysis of data regarding CO exposures, males represented an alarming $74 \%$ of unintentional non-fire related deaths and significant long-term morbidity, with an estimated $50 \%$ of neurologic sequelae in the non-fatal cases [8].

\section{Pathophysiology}

CO intoxication impairs oxygen delivery to tissues, resulting in cellular ischemia [18,19]. It is explained by the tetrameric structure of the hemoglobin molecule and the affinity among the heme groups when the CO is bound at one of its four sites, which causes a left shift of the oxygen-hemoglobin dissociation curve and a hyperbola-like shape [15]. The latter results in a hemoglobin molecule that has a decreased capacity to release oxygen to the tissues, including the respiratory center, leading to acidosis. CO causes mitochondrial dysfunction due to binding to the cytochrome c oxidase, leading to worsening of hypoxia, and increasing the production of reactive oxygen species by alteration of the electron transport chain $[19,20]$. In severe CO exposures (3000 ppm CO), the cAMP pathway has a deleterious role in ischemia-induced brain-damage [21].

\section{Clinical presentation}

The symptoms of CO poisoning are usually nonspecific and can imitate a wide variety of disorders [7,14]. Oxygen-dependent organs and those with the lowest metabolic stores show the initial signs of injury, such as the brain and heart [5]. The severity of CO poisoning depends on several aspects: exposure to this gas, being the most important, the concentration of molecules of $\mathrm{CO}$ in the air, and the observed level of $\mathrm{COHb}$. In acute poisonings, a concentration below 1000 parts per million (ppm) and a $\mathrm{COHb}$ of $10 \%$ typically results in headache; concentrations above 1000 ppm and COHb of 20\% results in dizziness, irritability, dyspnea, nausea, confusion, and syncope [22]. In patients with exposure from $3000 \mathrm{ppm}$ and more than $50 \%$ of $\mathrm{COHb}$, seizures, coma, and death may occur after prolonged exposure [23]. Myocardial injury and fibrosis have been reported after chronic exposure to moderate CO concentration; arrhythmias and sudden death (especially among patients with preexisting cardiac conditions) have been associated with acute environmental exposure (up to $500 \mathrm{ppm}$ ) [16]. It has been suggested that delayed CO encephalopathy can cause cognitive impairment and memory loss, and neuropsychologic symptoms, days to weeks after the exposure [24-26]. The classic cherry red hue that is commonly associated with CO poisoning patients is an uncommon postmortem sign and is rarely appreciated in the clinical assessment $[8,15]$.

\section{Diagnosis}

The diagnosis of COP rests in the high level of suspicion based on a detailed history of events, a key component of the initial assessment. Several diagnostic methods have been developed with the purpose of a rapid diagnosis, currently, the measurement of $\mathrm{COHb}$ by blood gas analysis represents the standard 
approach to a patient with a possible gas intoxication, several disadvantages of this method resulted from delayed blood assays and unavailability of first respondents in non-hospital scenarios have made clinicians look for other options for a timely diagnosis of the poisoning $[8,17,18,27]$. In recent studies, the efficacy of CO oximeters and exhaled CO analyzers during the initial assessment have been remarkable in diagnosing the most severe poisoning cases in very short periods of time [13,17,27].

\section{Review}

\section{Materials and methods}

Data Sources

This study protocol was prepared following PRISMA guidelines. We sought to identify all relevant manuscripts that compared COP treatment with $\mathrm{HBO}$ and $\mathrm{NBO}$. Our objective was to demonstrate the outcome with different therapeutic interventions. We searched electronic medical databases (PubMed ${ }^{\mathrm{TM}}$, ScienceDirect ${ }^{\mathrm{TM}}$, and Scielo ${ }^{\mathrm{TM}}$ ) and excluded studies that did not provide the outcome data. The authors independently searched relevant studies published from December 1989 to March 2017. All types of articles, including comprehensive reviews, prospective observational studies, randomized controlled trials (RCTs), multi-group comparison, editorials, retrospective studies, and case reports, were consulted for analysis of relevant content.

Data Search

The following keywords were utilized: hyperbaric oxygenation therapy, normobaric oxygenation therapy, carbon monoxide poisoning, carboxyhemoglobin, and Haldane effect. The articles were selected if they reported outcome data as well as complications, sequelae and/or mortality. The references quoted in the selected articles were also reviewed independently and added for further analysis and comparison between $\mathrm{HBO}$ and NBO outcomes. Issues regarding article eligibility for data collection were resolved by discussions with another member of the team.

\section{Results}

A total of 2124 articles were found from database searching and other sources and later scrutinized for any data related to carbon monoxide poisoning treatment with hyperbaric therapy and normobaric therapy (Figure 1). Only 16 clinical studies dealt specifically with outcomes after HBO/NBO therapy for COP. Twelve of them showed HBO as the therapeutic intervention of choice in COP; four studies showed that NBO was a reasonable method due to its cost-effectiveness and availability in the ED.

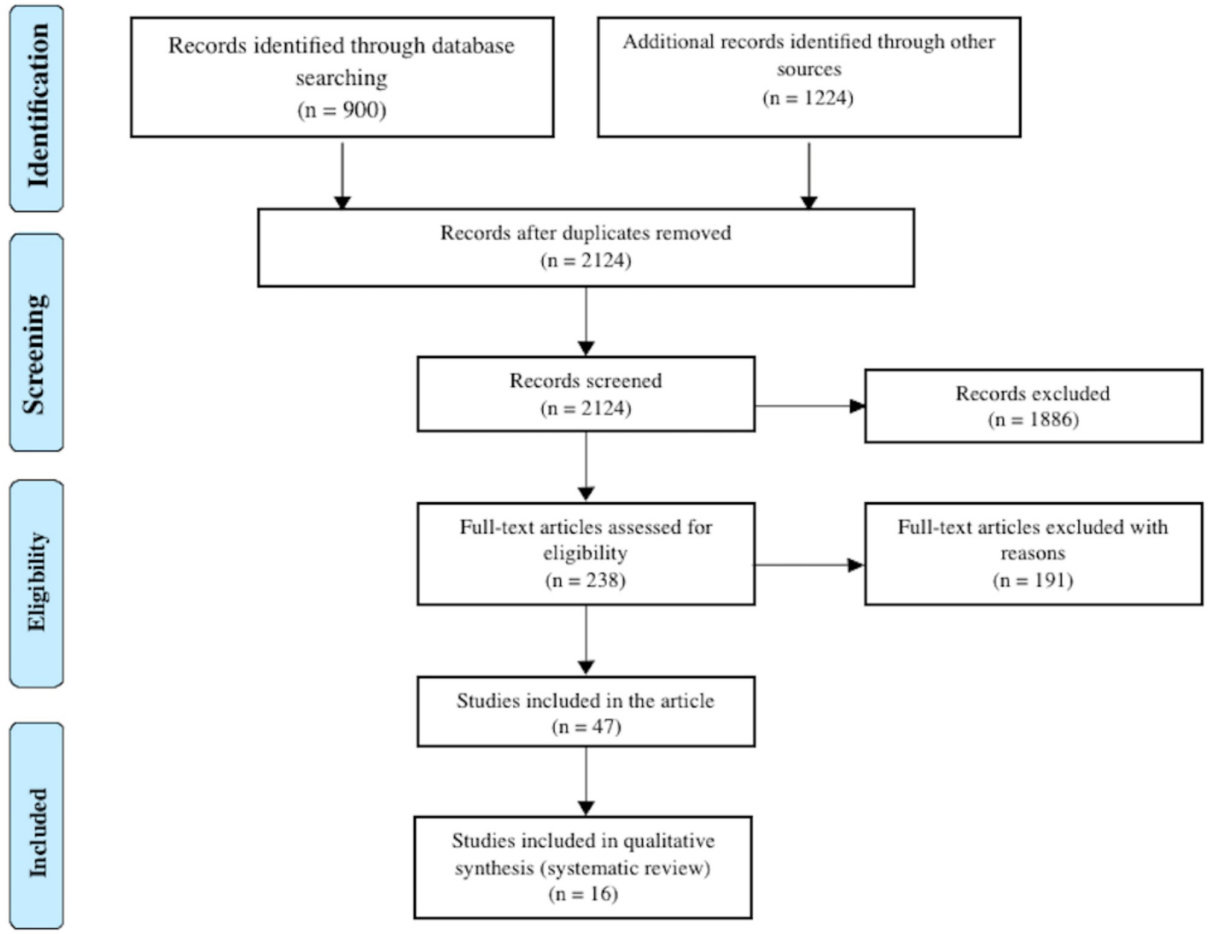

FIGURE 1: Flow diagram for study selection according to PRISMA 2009 guidelines 


\section{Discussion}

The highly unspecific clinical presentation of carbon monoxide poisoning (COP) entails a delayed diagnosis and treatment that represents an important impact on the probability of short and long-term neurological sequelae that are directly related to an early cognitive decline. In the last two decades, several studies have been focused on expedited diagnosis which in turn leads to prompt treatments and possibly better outcomes. The main controversy has been whether to use HBO or NBO. Historically, Henshaw in 1662, first applied compressed air, trying to prove the HBO theory but the experiment barely reached the pressure of $1.3 \mathrm{~atm}$ due to the inefficiency of his equipment [28]. This therapy elevates arterial and tissue oxygen tension, therefore, it enhances the elimination of carbon monoxide, meanwhile, increasing the adenosine triphosphate (ATP) production, reducing the oxidative stress and inflammation [7,8,29].

The half-life of the $\mathrm{COHb}$ is three to four hours when breathing room air, which is reduced to 30 to $90 \mathrm{~min}$ in the presence of $100 \%$ oxygen (normobaric oxygenation) and to 15 to 23 minutes with $\mathrm{HBO}$ at $2.5 \mathrm{~atm}$ [30]. The use of $\mathrm{HBO}$ has been demonstrated in previous studies that it significantly reduces the rate of complications, neurological sequelae, and mortality. In 1999, Camporesi demonstrated a decline in mortality from $30 \%$ to $13.5 \%$, in a group of 213 patients; none of the patients treated with HBO suffered from sequelae [31]. Garrabou et al. proved the effectiveness in recovering mitochondrial complex IV (mtCIV) function depends on the oxygen therapy that was administered [29]. Having said that, since HBO was more effective than normobaric oxygenation (NBO) in moderate and severe acute $\mathrm{COP}$, one session of $\mathrm{HBO}$ was effective enough to restore mitochondrial activity reducing the rate of possible complications [20,29,32].

Researches have concluded that the molecular effect of $\mathrm{HBO}$ results in the prevention of lipid peroxidation in the central nervous system and preservation of ATP levels in tissues exposed to carbon monoxide, therefore reducing the damage [33-35]. Evidence suggests that $\mathrm{HBO}$ can reduce neurological/cognitive sequelae of COP if administered within 24 hours of an acute episode of CO intoxication [36-38]. Vomero et al. analyzed three clinical cases of $\mathrm{CO}$ poisoning, in which the three patients were found unconscious; they mentioned that HBO must be applied under certain criteria such as loss of consciousness, neurological disturbance or coma [39]. This being the case, the patient must be transferred safely and hemodynamically stable $[40,41]$. A prospective trial demonstrated that the incidence of cognitive sequelae was lower in patients who underwent three HBO sessions within 24 hours after COP compared with patients treated with NBO $[8,33]$. The Undersea and Hyperbaric Medical Society, recommends HBO therapy for patients with serious $\mathrm{CO}$ poisoning as manifested by unconsciousness, abnormal neurologic signs, cardiovascular dysfunction such as myocardial infarction and acute coronary syndrome, severe acidosis or patients who are older than 36 years of age that were exposed for more than 24 hours, displaying a carboxyhemoglobin level of $25 \%$ or more. Thom and colleagues performed a randomized control trial on 60 patients (divided into two groups) with mild-moderate COP presented during the first six hours post-exposure. Neurologic sequelae were seen in $23 \%$ of patients in the control group and none in the HBO group [15,42]. Ducasse et al. performed a similar study in 26 non-comatose patients with acute COP divided into two groups and at $12 \mathrm{~h}$ none of the patients in the HBO group (0/13) had abnormal clinical findings, compared to the NBO group with 5/13 [43]. In 2017, Huang and colleagues showed that patients with CO poisoning who received HBO therapy had a lower mortality rate than those who did not, especially patients who were younger than 20 years and those with acute respiratory failure. In this study, patients under 20 years of age with acute respiratory failure had even more reduction in the mortality risk after $\mathrm{HBO}$ [44]. Also, a large-scale randomized controlled trial in Taiwan studied 25,000 patients with COP over a 13-year time frame; 7000 underwent $\mathrm{HBO}$ and they found improvement in mortality rate against those patients who underwent standard therapy (NBO) [45]. Several studies involving patients with different clinical settings suggested certain benefits in overall outcomes with the application of $\mathrm{HBO}$ at 2 or 3 ATA, and one clinical trial reporting superior outcomes with 3 ATA [9].

In 2015 the prevalence of CO exposure in pregnant women increased to $8.5 \%$ and this was associated with a mortality rate between $19 \%$ and $24 \%$ and a fetal mortality rate between $36 \%$ and $67 \%$ [5]. Friedman performed a review of literature among pregnant patients and concluded that HBO therapy is appropriate since these patients should generally require five times the length of the treatment in order to avoid sequelae in both, the mother and the fetus [5].

NBO hastens the elimination of carbon monoxide and is safe, easily available and inexpensive. If used as a single-agent treatment, it should be provided until the carboxyhemoglobin level is less than $5 \%$. Evidence suggests that NBO may be less effective than $\mathrm{HBO}$ for preventing cognitive sequelae. A prospective study showed that $34 \%$ of NBO-treated patients reported symptoms such as headaches or memory problems at four weeks and $46 \%$ of the patients had neuropsychological sequelae at six weeks [7]. Another study showed that NBO was the appropriate therapy, even though the patients developed neurological symptoms at about six weeks after treatment; it also suggested that the treatment with $\mathrm{HBO}$ has been a controversial issue, and precise guidelines must be established in order to be applied [46]. Sen et al. established that the treatment of $\mathrm{CO}$ intoxication first requires a high-flow of $100 \% \mathrm{NBO}$ until $\mathrm{COHb}$ is normalized [3]. Another study performed by Scheinkestel refuted the use of $\mathrm{HBO}$ in $\mathrm{CO}$ poisoning; they provided a daily 60 minute treatment at 2.8 ATA on three consecutive days for the HBO group in conjunction with prolonged high flow oxygen therapy between treatments (this group received oxygen therapy equated to approximately 35.7 


\section{Cureus}

$\mathrm{COHb}$-dissociation half-lives, while the $\mathrm{NBO}$ group received the equivalent of $28.5 \mathrm{COHb}$-dissociation halflives), while the NBO group received at least three consecutive days continuous oxygen by non-occlusive facemask at $14 \mathrm{~L} / \mathrm{min}$ [47]. The authors attributed the worse outcome in the HBO group to the higher doses of oxygen that may add no further benefit and possibly causing adverse effects. This study had a low follow up rate (only $46 \%$ of patients attended the one month follow up). We excluded this paper from further analysis since no clinical evaluation was assessed to rule out sequelae or for any clinical outcome in both groups [47].

The management of $\mathrm{CO}$ poisoning eliminates carbon monoxide from the body and increases the oxygen content of the blood by the means of administration of high flow and concentration of oxygen, ventilatory support, and monitoring for abnormal cardiac rhythms [7,15]. Most of the studies in this review showed that $\mathrm{HBO}$ had greater therapeutic efficacy over NBO (Table 1). HBO was appropriate for life-threatening CO poisoning with $\mathrm{COHb}$ levels greater than $15 \%$, and in patients with a history of loss of consciousness, neurological symptoms, pregnancy or cardiac compromise [5]. The disadvantages of HBO therapy included barotrauma, hypoxic seizures due to high intake of oxygen in a short period of time and those risks associated with the transport of patients to treatment centers $[8,34,37]$. 


\section{Cureus}

Studies supporting the effectiveness of HBO

Article $\quad \begin{aligned} & \text { Type of } \\ & \text { study }\end{aligned}$ Comments

Reduce the rate of spontaneous abortion in pregnancy

Koren et

al. $1991[6]$

PS with $\mathrm{CO}$ poisoning. In two Cases were $\mathrm{COHb}$ was $39 \%$ and $21 \%$, HBO was applied and normal outcome was seen in the 1 st year of life.

Jang et al. PS Improved mitochondrial dysfunction.
2017 [20]

Garrabou

et al. 2011

[29]

Camporesi

1999 [31]

ROL

$30 \%$

Jurič et al.

2015 [32]

MGC

HBO reduced toxic effects of $\mathrm{CO}$ in astrocytes while NBO showed no beneficial effect.

Weaver et

al. 2002 RCT Reduced the cognitive sequelae by $46 \%$.

[33]

Perez et

al. 2017

[35]

Santiago I.

2003 [37]

ROL

Lueken et

al. 2006

CR

[38]

Thom et

al. 1995

[42]

Ducasse

et al. 1995 RCT

[43]

Huang et

al. 2017

RCS

[44]

The only effective treatment to avoid delayed neuropathy,
Studies supporting the effectiveness of NBO

Article $\begin{aligned} & \text { Type of Comments } \\ & \text { study }\end{aligned}$

administered until $\mathrm{COHb}$

Sen et al. CR is normalized, since

2010 [3] CR HBO usage is

controversial.

High-flow $100 \%$ oxygen

Weaver L. CR/ROL is safe, available and

2009 [7] CR/ROL inexpensive compared to $\mathrm{HBO}$.

Bor-

NBO caused a

decrement in red blood

Kucukatay MGC cells aggregation, HBO

et al. 2010 caused increment of free

Bartolome

et al. 2010 ROL

[46]

radical production.

NBO is the treatment for election with the proper follow up.

\section{TABLE 1: HBO and NBO studies comparison}

Abbreviations: HBO, hyperbaric oxygenation; NBO normobaric oxygenation; ROL, review of literature; PS, prospective study; MGC, multi-group comparison; RCT, randomized control trial; $\mathrm{CR}$, case report; RCS, retrospective cohort study; CO, carbon monoxide; $\mathrm{COHb}$, carboxyhemoglobin; ACOP, acute carbon monoxide poisoning

\section{Conclusions}

Our systematic analysis showed that HBO is effective in moderate to high-risk situations and should be the therapy of choice to avoid sequelae, especially neurologically. This type of treatment has been useful in the last decades due to its powerful effect to counteract the toxicity of carbon monoxide as it decreases the half- 
life of carboxyhemoglobin in the blood-stream considerably, reducing it to less than a quarter of its normal physiological half-life. It is widely known that not every medical center has hyperbaric chambers due to its cost and expertise, therefore, NBO can be considered as a viable therapeutic option, presenting acceptable outcomes in comparison with $\mathrm{HBO}$.

\section{Additional Information \\ Disclosures}

Conflicts of interest: In compliance with the ICMJE uniform disclosure form, all authors declare the following: Payment/services info: All authors have declared that no financial support was received from any organization for the submitted work. Financial relationships: All authors have declared that they have no financial relationships at present or within the previous three years with any organizations that might have an interest in the submitted work. Other relationships: All authors have declared that there are no other relationships or activities that could appear to have influenced the submitted work.

\section{Acknowledgements}

This study protocol was prepared following PRISMA guidelines. PROSPERO does not currently accept registrations for scoping reviews, literature reviews or mapping reviews.

\section{References}

1. Jung Y, Lee J, Min Y, et al.: Carbon monoxide-induced cardiomyopathy. Circ J. 2014, 78:1437-1444. 10.1253/circj.cj-13-1282

2. Johnson-Arbor K, Quental A, Li D: A comparison of carbon monoxide exposures after snowstorms and power outages. Am J Prev Med. 2014, 46:481-486. 10.1016/j.amepre.2014.01.006

3. Sen S, Peltz C, Beard J, Zeno B: Recurrent carbon monoxide poisoning from cigarette smoking . Am J Med Sci. 2010, 340:427-428. 10.1097/MAJ.0b013e3181ef712d

4. De Juniac A, Kreis I, Ibison J, Murray V: Epidemiology of unintentional carbon monoxide fatalities in the UK. Int J Environ Health Res. 2011, 22:210-219. 10.1080/09603123.2011.628645

5. Friedman P, Guo X, Stiller R, Laifer S: Carbon monoxide exposure during pregnancy. Obstet Gynecol Surv. 2015, 70:705-712. 10.1097/OGX.0000000000000238

6. Koren G, Sharav T, Pastuszak A, et al.: A multicenter, prospective study of fetal outcome following accidental carbon monoxide poisoning in pregnancy. Reprod Toxicol. 1991, 5:397-403. 10.1016/08906238(91)90002-W

7. Weaver L: Carbon monoxide poisoning. N Engl J Med. 2009, 360:1217-1225. 10.1056/NEJMcp0808891

8. Lippi G, Rastelli G, Meschi T, Borghi L, Cervellin G: Pathophysiology, clinics, diagnosis and treatment of heart involvement in carbon monoxide poisoning. Clin Biochem. 2012, 45:1278-1285. 10.1016/i.clinbiochem.2012.06.004

9. Annane D, Chadda K, Gajdos P, Jars-Guincestre MC, Chevret S, Raphael JC: Hyperbaric oxygen therapy for acute domestic carbon monoxide poisoning: two randomized controlled trials. Intensive Care Med. 2011, 37:486-492. 10.1007/s00134-010-2093-0

10. Raphael JC, Elkharrat D, Jars-Guincestre MC, et al.: Trial of normobaric and hyperbaric oxygen for acute carbon monoxide intoxication. Lancet. 1989, 334:414-419. 10.1016/S0140-6736(89)90592-8

11. Caviedes I, Borzone G, Briceño C, et al.: Standardization of the single-breath diffusing capacity. Chilean Society of Respiratory Diseases guidelines (Article in Spanish). Rev chil enferm respir. 2014, 30:145-155. 10.4067/S0717-73482014000300004

12. Varlet V, Lagroy E, Augsburger M, Mangin P: A new approach for the carbon monoxide (CO) exposure diagnosis: measurement of total CO in human blood versus carboxyhemoglobin (HbCO). J Forensic Sci. 2013, 58:1041-1046. 10.1111/1556-4029.12130

13. Hullin T, Aboab J, Desseaux K, Chevret S, Annane D: Correlation between clinical severity and different non-invasive measurements of carbon monoxide concentration: a population study. PLoS One. 2017, 12:1-9. 10.1371/journal.pone.0174672

14. Prockop L, Chichkova R: Carbon monoxide intoxication: an updated review. J Neurol Sci. 2007, 262:122-130. 10.1016/j.jns.2007.06.037

15. Varon J, Marik P, Fromm R, Gueler A: Carbon monoxide poisoning: a review for clinicians . J Emerg Med. 1999, 17:87-93. 10.1016/S0736-4679(98)00128-0

16. Dallas M, Yang Z, Boyle J, et al.: Carbon monoxide induces cardiac arrhythmia via induction of the late $\mathrm{Na}^{+}$ current. Am J Respir Crit Care Med. 2012, 186:648-656. 10.1164/rccm.201204-06880C

17. Sebbane M, Claret P, Mercier G, et al.: Emergency department management of suspected carbon monoxide poisoning: role of pulse CO-oximetry. Respir Care. 2013, 58:1614-1620. 10.4187/respcare.02313

18. Sibon A, Martinez P, Vizcaya M, Romero JL: Poisoning by carbon monoxide (Article in Spanish) . Cuad Med Forense. 2007, 13:65-69. 10.4321/S1135-76062007000100007

19. Guzman J: Carbon monoxide poisoning. Crit Care Clin. 2012, 28:537-548. 10.1016/j.ccc.2012.07.007

20. Jang DH, Kelly M, Hardy K, Lambert DS, Shofer FS, Eckmann DM: A preliminary study in the alterations of mitochondrial respiration in patients with carbon monoxide poisoning measured in blood cells. Clin Toxicol (Phila). 2017, 55:579-584. 10.1080/15563650.2017.1288912

21. Hara S, Mizukami H, Kuriiwa F, Mukai T: cAMP production mediated through P2Y11-like receptors in rat striatum due to severe, but not moderate, carbon monoxide poisoning. Toxicology. 2011, 288:49-55. 10.1016/j.tox.2011.07.001

22. Sandilands E, Bateman D: Carbon monoxide. Medicine. 2016, 44:151-152. 10.1016/j.mpmed.2015.12.024

23. Aldossary M, Almadni O, Kharoshah M, Alsaif D, Alsowayigh K, Alfaraidy M: Carbon monoxide toxicity in 
Dammam, KSA: retrospective study. Egypt J Forensic Sci. 2015, 5:36-38. 10.1016/j.ejfs.2014.10.002

24. Ochi S, Abe M, Li C, et al.: The nicotinic cholinergic system is affected in rats with delayed carbon monoxide encephalopathy. Neurosci Lett. 2014, 569:33-37. 10.1016/j.neulet.2014.03.054

25. Tormoehlen L: Toxic leukoencephalopathies. Neurol Clin. 2011, 29:591-605. 10.1016/j.ncl.2011.05.005

26. Weaver L, Deru K: Carbon monoxide poisoning at motels, hotels and resorts . Am J Prev Med. 2007, 33:23-27. 10.1016/j.amepre.2007.02.038

27. Piatkowski A, Ulrich D, Grieb G, Pallua N: A new tool for the early diagnosis of carbon monoxide intoxication. Inhal Toxicol. 2009, 21:1144-1147. 10.3109/08958370902839754

28. Jain KK: The history of hyperbaric medicine. Textbook of Hyperbaric Medicine. Jain KK (ed): Springer, Cham, Kirkland, WA; 2017. Sixth edition:3-9. 10.1007/978-3-319-47140-2_1

29. Garrabou G, Inoriza J, Moren C, et al.: Mitochondrial injury in human acute carbon monoxide poisoning: the effect of oxygen treatment. J Environ Sci Health C Environ Carcinog Eco Toxicol Rev. 2011, 29:32-51. 10.1080/10590501.2011.551316

30. Weaver LK, Howe S, Hopkins R, Chan KJ: Carboxyhemoglobin half-life in carbon monoxide-poisoned patients treated with 100\% oxygen at atmospheric pressure. Chest. 2000, 117:801-808. 10.1378/chest.117.3.801

31. Camporesi E: Hyperbaric oxygen therapy: applications in the trauma patient . Anesthesiol Clin North Am. 1999, 17:311-323. 10.1016/S0889-8537(05)70095-X

32. Jurič D, Finderle Ž, Šuput D, Brvar M.: The effectiveness of oxygen therapy in carbon monoxide poisoning is pressure- and time-dependent: a study on cultured astrocytes. Toxicol Lett. 2015, 233:16-23. 10.1016/j.toxlet.2015.01.004

33. Weaver L, Hopkins R, Chan K, et al.: Hyperbaric oxygen for acute carbon monoxide poisoning . N Engl J Med. 2002, 347:1057-1067. 10.1056/NEJMoa013121

34. Bor-Kucukatay M, Atalay H, Karagenc N, Erken G, Kucukatay V: The effect of carbon monoxide poisoning on hemorheological parameters in rats and the alterations in these parameters in response to three kinds of treatments. Clin Hemorheol Microcirc. 2010, 44:87-96. 10.3233/CH-2010-1254

35. Pérez TJG, Bautista AMI, Moranchel GL, Martiñón RR, Hernández AM: Síndrome coronario agudo secundario a intoxicación por monóxido de carbono y respuesta al tratamiento con oxígeno hiperbárico (Article in Spanish). Acta méd. Grupo Ángeles. 2017, 15:230-233.

36. Hampson N, Little C: Hyperbaric treatment of patients with carbon monoxide poisoning in the United States. Undersea Hyperb Med. 2005, 32:21-26.

37. Santiago I: Gas poisoning (Article in Spanish). Anales Sis San Navarra. 2003, 26:173-180. 10.4321/S113766272003000200010

38. Lueken R, Heffner A, Parks P: Treatment of severe carbon monoxide poisoning using a portable hyperbaric oxygen chamber. Ann Emerg Med. 2006, 48:319-322. 10.1016/j.annemergmed.2006.01.014

39. Vomero A, Pandolfo S, Vasquez M, Mas M, Bello O: Intoxicación por monóxido de carbono. Análisis de tres casos clínicos (Article in Spanish). Arch Pediatr Urug. 2009, 80:204-209.

40. Hampson NB, Bodwin D: Toxic CO-ingestions in intentional carbon monoxide poisoning . J Emerg Med. 2013, 44:625-630. 10.1016/j.jemermed.2012.08.033

41. Duenas-Laita A, Burillo P, Alonso JR, et al.: Basis for the clinical management of fire smoke poisoning "docohumo Madrid 2010" (Article in Spanish). Med Intensiva. 2010, 34:609-619. 10.1016/j.medin.2010.07.007

42. Thom S, Taber R, Mendiguren II, Clark JM, Hardy KR, Fisher AB: Delayed neuropsychologic sequelae after carbon monoxide poisoning: prevention by treatment with hyperbaric oxygen. Ann Emerg Med. 1995, 25:474-480. 10.1016/S0196-0644(95)70261-X

43. Ducasse JL, Celsis P, Marc-Vergnes JP: Non-comatose patients with acute carbon monoxide poisoning: hyperbaric or normobaric oxygenation?. Undersea Hyperbaric Med. 1995, 22:9-15.

44. Huang CC, Ho CH, Chen YC, et al.: Hyperbaric oxygen therapy is associated with lower short- and long-term mortality in patients with carbon monoxide poisoning. Chest. 2017, 152:943-953. 10.1016/j.chest.2017.03.049

45. Cowl CT: Justifying hyperbaric oxygen delivery for carbon monoxide poisoning. Chest. 2017, 152:911-913. 10.1016/j.chest.2017.07.022

46. Bartolome T, Amores P, Cuesta E, Gallego N: Carbon monoxide poisoning: an undervalued event in the emergency department (Article in Spanish). Rev Clin Med Fam. 2010, 3:220-222.

47. Scheinkestel CD, Bailey M, Myles PS, et al.: Hyperbaric or normobaric oxygen for acute carbon monoxide poisoning: a randomised controlled clinical trial. Med J Aust. 1999, 170:203-210. 10.5694/j.13265377.1999.tb140318.x 